\title{
Transverse propagation of ultraviolet and infrared femtosecond laser pulses in photonic crystal fibers
}

\author{
Tigran Baghdasaryan, ${ }^{* 1}$ Thomas Geernaert, ${ }^{1}$ Pawel Mergo, ${ }^{2}$ Francis Berghmans ${ }^{1}$ and Hugo Thienpont ${ }^{1}$ \\ ${ }^{1}$ Brussels Photonics Team B-PHOT, Vrije Universiteit Brussel (VUB), Pleinlaan 2, 1050 Brussels, Belgium \\ ${ }^{2}$ Maria Curie-Skłodowska University, Pl. Marii Curie-Skłodowskiej 5, 20-031 Lublin, Poland
}

Received May 19, 2012; accepted June 19, 2012; published June 30, 2012

\begin{abstract}
We studied the influence of angular orientation of a photonic crystal fiber (PCF) on optical power that reaches the fiber core during femtosecond grating inscription at $267 \mathrm{~nm}$ and $800 \mathrm{~nm}$. In our numerical study we considered two types of PCFs: three PCF designs with a standard hexagonal lattice and one highly birefringent PCF with three rows of air holes. A dedicated figure of merit, the Transverse Coupling Efficiency (TCE), is used throughout the paper to estimate the influence of the PCF cladding on laser power that reaches the core region. We found that at an inscription laser wavelength of $800 \mathrm{~nm}$ and for a high filling factor hexagonal lattice PCF, the TCE can reach 1.4 This means that the microstructure can actually help deliver higher power to the core region of the PCF than what can be achieved with a conventional step-index fiber of similar diameter. For a highly birefringent PCF even higher TCE values (up to 2.8) can be achieved for a wide range of orientations.
\end{abstract}

Fiber Bragg gratings (FBGs) are well known as essential elements in optical communications where they can serve for filtering, multiplexing, dispersion compensation, etc [1]. FBGs have also found applications as cavity mirrors in all-fiber lasers [2]. Another popular application field for FBGs is optical sensing, where they can be used for temperature, strain and humidity sensing and as well as in more advanced structural health monitoring systems [3].

Conventional grating inscription methods in step-index fibers exploit the photosensitivity of a doped fiber core to ultraviolet (UV) light. An interference pattern of two laser beams then induces periodic modulation of the refractive index in the core [1]. More recent methods for grating inscription use femtosecond pulse lasers at both UV and IR wavelengths. Owing to the high peak power in short laser pulses, the refractive index change can even be induced in non-photosensitive fibers via one or more multi-photon absorption processes [4]. Harmonics of a Ti:Sapphire laser at UV 267nm [5] and IR 800nm [6] are commonly used for that purpose. In the first case the refractive index change is induced by a two photon absorption process, while in the latter it involves a five photon absorption process [4].

Femtosecond gratings can be successfully inscribed in conventional optical fibers, but attempting to do so in photonic crystal fibers (PCFs) is not straightforward. In the case of PCFs the inscribing beams have to pass

*E-mail: tbaghdas@b-phot.org through the holey structure before reaching the core. The multiple air-silica interfaces tend to impede sufficient laser power delivery to the core [6]. As a result grating inscription is either less efficient or simply not achievable. The inscription of femtosecond point-by-point gratings in PCFs, for example, has proven to be highly problematic [7-8].

In recent years several authors have studied how the PCF microstructure affects transverse propagation of laser light on its way to the core [9-11]. The detrimental influence of the holey structure was repeatedly reported for several commercially available PCFs. The angular orientation of the PCF relative to the direction of the inscribing beam was also found to greatly influence the grating growth [11].

In this letter we deal with this issue as well and we report on a numerical study of the influence of the PCF microstructure on the transverse coupling of pulsed laser power to the PCF core region. We target grating inscription applications at two wavelengths: $267 \mathrm{~nm}$ and $800 \mathrm{~nm}$. For our study we considered a standard hexagonal lattice PCF and a highly birefringent PCF with only three rows of air holes. This last PCF was used previously for the inscription of a point-by-point grating [7].

The letter is structured as follows. First we introduce the transverse coupling efficiency (TCE) as a figure of merit. We then analyze how the TCE varies as a function of the angular orientation of the PCF and we do so for three hexagonal lattice PCFs with different filling factors. The next section conducts a similar analysis for the highly birefringent PCF.

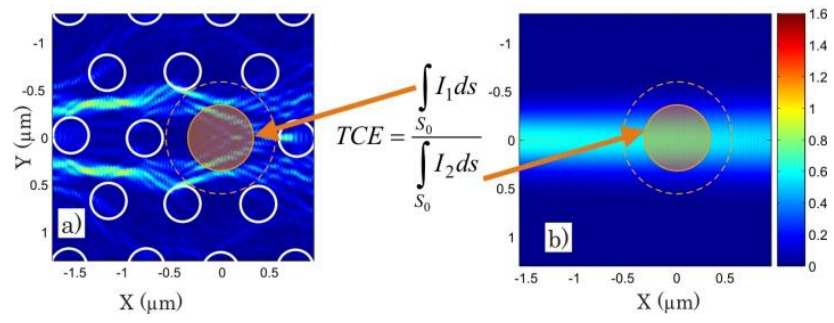

Fig. 1. Calculation of the transverse coupling efficiency based on the ratio of the optical power in the core region for a fiber with and without microstructure. 
We start by introducing the figure of merit that is used throughout the letter to estimate the influence of the PCF microstructure on the amount of laser pulse power that reaches the core region. We define transverse coupling efficiency (TCE) as a ratio of integrated core field intensities in the presence and in the absence of the PCF microstructure. Fig. 1 illustrates the way of calculating this TCE. Fig. 1a shows the intensity distribution in the core region as obtained from a simulation carried out with the commercially available software Lumerical FDTD Solutions. This distribution is integrated over a circular area in the core region. The same calculation is carried out for a structure without air holes (Fig. 1b) and the ratio of the integrals yields the TCE (see [12] for more details). We used an incident beam with a transverse Gaussian profile and a $15 \mu \mathrm{m}$ waist radius. The femtosecond pulses at $267 \mathrm{~nm}$ and $800 \mathrm{~nm}$ have duration of $350 \mathrm{fs}$ and $125 \mathrm{fs}$, respectively. These are all characteristic values when working with a Ti:Sapphire laser in a fs grating inscription setup.

First we studied the dependence of the TCE on the orientation of the fiber for three hexagonal lattice PCFs. All the PCFs have the same 6 ring structure but have different air hole filling factors. The holey structure of those PCFs is shown in Fig. 2; the air hole pitch is $\Lambda=3.46 \mu \mathrm{m}$, while the air hole radius values are $\mathrm{R}=0.68 \mu \mathrm{m}, \mathrm{R}=1.1 \mu \mathrm{m}$ and $\mathrm{R}=1.5 \mu \mathrm{m}$. The results obtained for those different PCFs allow estimating the influence of the filling factor on the TCE as well.

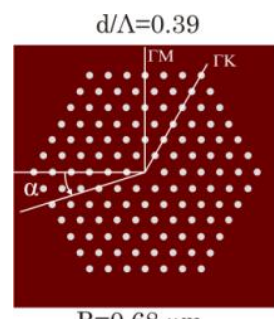

$\mathrm{R}=0.68 \mu \mathrm{m}$

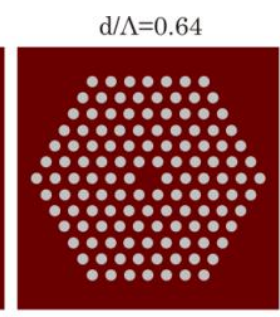

$\mathrm{R}=1.1 \mu \mathrm{m}$

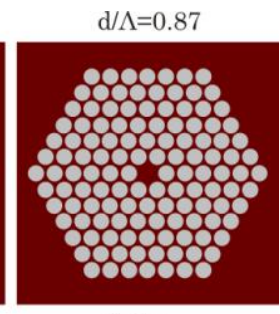

$\mathrm{R}=1.5 \mu \mathrm{m}$
Fig. 2. Cross-section lay-outs of the three hexagonal lattice PCFs under study. $\alpha$ indicates the orientation angle with respect to the incident laser beam.

The results of the TCE dependence on the PCF orientation are presented in Fig. 3. For reasons of symmetry the simulations are performed for orientation angles from $\alpha=0^{\circ}$ ( $\Gamma \mathrm{K}$ direction of hexagonal lattice) to $30^{\circ}(\Gamma \mathrm{M})$ only, in increments of $1^{\circ}$. Figure 3 a depicts the results for an incident beam at $267 \mathrm{~nm}$. Because the TCE is lower than 1 for all three PCFs at all orientations, these hexagonal microstructures have a detrimental influence on optical power transversely coupled to the core. The average value of the TCE is around 0.43 and varies between 0.24 and 0.72 . In addition, the features of the TCE curves are very similar for all three PCFs, which indicates that the filling factor does not affect the PCF transverse coupling properties at this wavelength.

For an incident beam at $800 \mathrm{~nm}$ the dependence of the TCE on the orientation is much more pronounced, as depicted in Fig. 3b. TCE values larger than 1 are found for the high filling factor PCF oriented at $30^{\circ}$. This result shows that for a dedicated design and for a particular orientation, the PCF microstructure can have a positive influence on the transverse coupling and, by the same token, on the efficiency of fs inscription of fiber gratings.

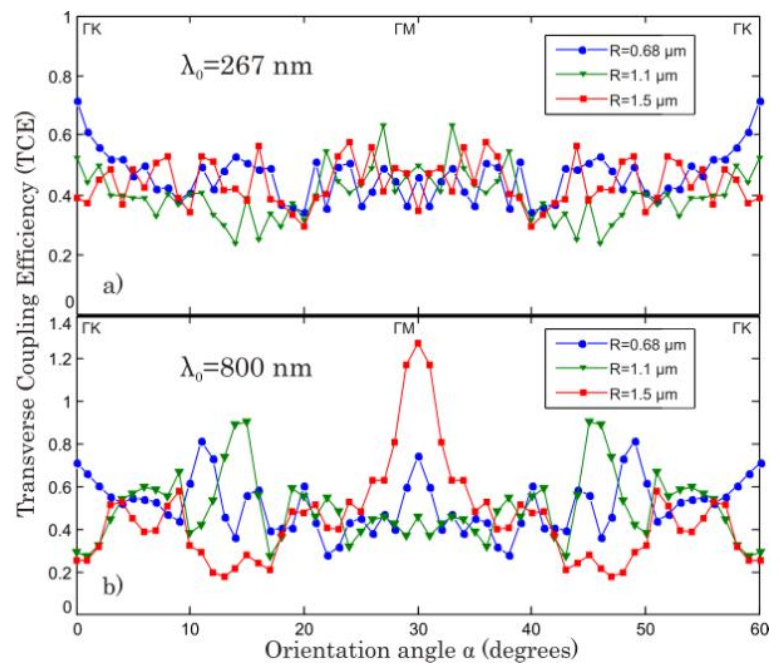

Fig. 3. TCE versus fiber orientation for the three hexagonal lattice PCFs under study. a) Results for $267 \mathrm{~nm}$ and b) Results for $800 \mathrm{~nm}$.

At $800 \mathrm{~nm}$, the average TCE is around 0.5. Therefore, when the orientation is not controlled, half the incident optical power - on average - will not reach the core. The largest TCE swing is detected for the high filling factor PCF $(\mathrm{R}=1.5 \mu \mathrm{m})$ : the minimum value is 0.19 at $13^{\circ}$ and a maximum value of 1.27 is found at $30^{\circ}$.

We now deal with a highly birefringent PCF with three rows of air holes shown in Fig. 4. This PCF was initially designed to facilitate conventional UV laser based FBG inscription [13]. It has also been used to fabricate the first point-by-point grating in a highly birefringent PCF [7]. In our simulations we used the actual cross-section of the PCF as obtained from a scanning electron microscope (SEM), instead of an ideal structure. This allows accounting for deviations from the design values of the hole pitch and hole radii that result from manufacturing imperfections.

Simulations were performed along the same scheme as for the hexagonal lattice PCFs. The only difference is that orientation angles from $0^{\circ}$ to $90^{\circ}$ were considered due to the symmetry of this particular PCF. It is also worthwhile noting that here the simulation area included the cladding region. Additional focusing influence of the outer cladding is thus taken into account in the simulations. 


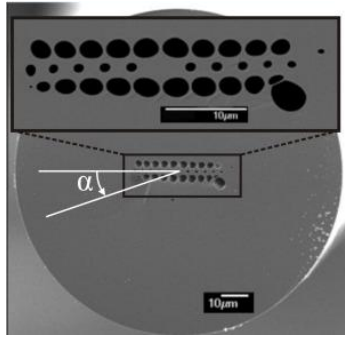

Fig. 4. Scanning electron microscope photograph of a highly birefringent PCF. $\alpha$ indicates the orientation angle with respect to the incident laser beam.

The evolution of the TCE with the orientation for $267 \mathrm{~nm}$ and $800 \mathrm{~nm}$ laser pulse wavelengths is shown in Fig. 5. Since at $90^{\circ}$ orientation the light needs to pass only one row of air holes before reaching the core region one can expect to find large TCE values at those orientations. We used the time averaged field intensity calculations as previously described to extract the results depicted in Fig. 5. These take into account multiple reflections and possible field oscillations in the core region. Because of the peculiar design of a highly birefringent PCF those effects - in particular laser pulse back-reflection from the second row of large air holes - are also responsible for higher TCE values around $90^{\circ}$ (see [11]).

At orientations of $0^{\circ}$ and $180^{\circ}$, the light needs to pass a larger numbers of air holes, which results in lower TCE values.

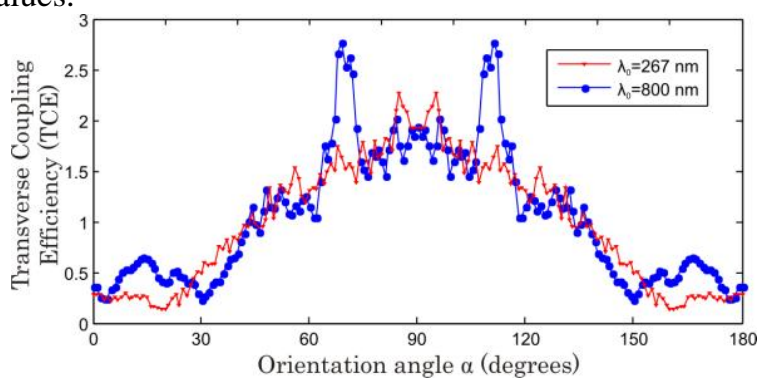

Fig. 5. TCE versus fiber orientation for the highly birefringent PCF.

It is noteworthy that high TCE values are detected around $90^{\circ}$ for both inscription wavelengths. At $800 \mathrm{~nm}$ the maximal TCE reaches 2.8 , whereas at $267 \mathrm{~nm}$ the maximal TCE value is 2.3. For this PCF and in a wide range of orientations (ranging from $45^{\circ}$ to $135^{\circ}$ ) the influence of the holey microstructure is only positive and allows increasing the optical power coupled to the core by more than $100 \%$.

Unlike hexagonal lattice PCFs the behaviour of the orientation dependence at $267 \mathrm{~nm}$ and $800 \mathrm{~nm}$ is very similar. In both cases an optimal coupling is not obtained at $90^{\circ}$ exactly, but around that value, as evidenced by the two peaks in the curves in Fig. 5. This can be tentatively explained by resonant oscillation of the light between two big air holes at opposite corners of the core region. At $800 \mathrm{~nm}$ the double peak nature is more pronounced and the distance between the peaks is larger. For the $267 \mathrm{~nm}$ wavelength pulse the TCE peaks are found at $84^{\circ}$ and $96^{\circ}$, while at $800 \mathrm{~nm}$ the TCE peaks are at $79^{\circ}$ and $111^{\circ}$.

In conclusion we have presented a numerical study on the transverse coupling of femtosecond laser pulses to the core of a PCF, as a function of angular orientation of the PCF relative to the laser beam. We have compared the transverse coupling at wavelengths of $267 \mathrm{~nm}$ and $800 \mathrm{~nm}$, which are wavelengths of choice for femtosecond grating inscription. To do so we used a figure of merit that we named the Transverse Coupling Efficiency (TCE). We first considered three hexagonal lattice PCFs with different air filling factors. At $267 \mathrm{~nm}$ we did not identify any peculiar feature in the TCE orientation dependence. However at $800 \mathrm{~nm}$ we found TCE values in excess of 1 for the highest filling factor PCF at an orientation of $30^{\circ}$ (corresponding to the $\Gamma \mathrm{M}$ direction of the hexagonal lattice). For the highly birefringent PCF, very high TCE values of 2.3 and 2.8 were detected for $267 \mathrm{~nm}$ and $800 \mathrm{~nm}$ respectively. At the same time the TCE orientation dependence for those two wavelengths at other orientations was nearly identical. This study indicates that careful control of the orientation of the PCF, dedicated design of microstructure features and adequate choice of the inscription wavelength may significantly increase the efficiency with which femtosecond gratings can be fabricated in PCFs.

This work was financially supported by the European Commission (EU) FP7 project "PHOSFOS" - Photonic Skins for Optical Sensing and by the COST TD1001 action "OFSESA". The authors also wish to acknowledge the Research Foundation-Flanders (FWO), the Methusalem and Hercules Foundations Flanders, as well as the Interuniversity Attraction Poles (IAP) - Belgian Science Policy.

\section{References}

[1] A. Othonos, K. Kalli, Fiber Bragg Gratings (Artech House 1999).

[2] W. Wadsworth, R. Percival, G. Bouwmans, J. Knight, P. Russell, Opt. Expr. 11, 48 (2003)

[3] Nature Photonics, Optical-fibre Sensors, Tech. Focus 2, 158 (2008)

[4] D.N. Nikogosyan, Meas. Sci. Tech. 18, R1 (2007).

[5] M. Becker, J. Bergmann, S. Brückner, M. Franke, E. Lindner, M.W. Rothhardt, H. Bartelt, Opt. Expr. 16, 19169 (2008).

[6] S.J. Mihailov, D. Grobnic, H. Ding, C.W. Smelser, J. Broeng, IEEE Photon. Technol. Lett. 18, 1837 (2006).

[7] T. Geernaert, K. Kalli, C. Koutsides, M. Komodromos, T. Nasilowski, W. Urbanczyk, et al., Opt. Lett. 35, 1647 (2010).

[8] T. Allsop, K. Kalli, K. Zhou, G.N. Smith, M. Komodromos, J. Petrovic, et al., J. Opt. Soc. Am. B 28, 2105 (2011).

[9] G.D. Marshall, D.J. Kan, A.A. Asatryan, L.C. Botten, M.J. Withford, Opt. Expr. 15, 7876 (2007).

[10] S. Pissadakis, M. Livitziis, G. Tsibidis, J. Eur. Opt. Soc. Rapid Publ. 4, 09049 (2009).

[11] T. Baghdasaryan, T. Geernaert, M. Becker, K. Schuster, H. Bartelt M. Makara, et al., IEEE Phot. Tech. Lett. 23, 1832 (2011).

[12] T. Baghdasaryan, T. Geernaert, F. Berghmans, H. Thienpont, Opt. Expr. 19, 7705 (2011)

[13] T. Geernaert, T. Nasilowski, K. Chah, M. Szpulak, J. Olszewski, G. Statkiewicz, et al., IEEE Phot. Tech. Lett. 20, 554 (2008). 